\title{
Doctors of the Movement System - Identity by Choice or Therapists Providing Treatment - Identity by Default
}

\author{
Shirley Sahrmann, PT, PhD, FAPTA \\ ${ }^{1}$ Washington University \\ Keywords: human movement, physical therapy \\ https://doi.org/10.26603/001c.30175
}

International Journal of Sports Physical Therapy

Vol. 17, Issue 1, 2022

\section{THE CHRONIC NATURE OF HEALTH CONDITIONS AND THE IMPLICATIONS FOR PHYSICAL THERAPY}

Two major developments in the understanding of the nature of health conditions should position physical therapy as a growth profession. One understanding is recognition that many, if not most, health conditions are chronic in nature. Conditions such as diabetes, cardiovascular disease and even some forms of cancer begin insidiously many years before the onset of symptoms and then continue throughout life. Lack of physical activity contributes to these conditions. ${ }^{1}$ These systemic conditions as well as musculoskeletal problems have their roots in lifestyle. ${ }^{1}$ The second development is the recognition of the importance of movement. As would be expected increased activity or exercise, is an effective intervention for health conditions ranging from cancer to dementia. ${ }^{2-6}$ The physical therapist should be considered as the health professional with the most comprehensive expertise in physical activity. Optimizing physical activity for health is more than just participating in a form of exercise. Physical activity should be focused on optimizing the components of movement, including the musculoskeletal and neuromuscular systems (including alignment and neuromuscular activation patterns) as well as the cardiovascular, pulmonary, and metabolic systems. In other words, optimizing the performance of the movement system. Physical activity including exercise should contribute to prevention or minimization of musculoskeletal problems because the recommended programs incorporate knowledge of structural variations, optimal movement patterns, and appropriate progression. That knowledge should be applied to analysis of specific activities such as running, biking, dance, and various sports.

\section{IS THE PHYSICAL THERAPIST CONSIDERED AS AN EXPERT IN MOVEMENT AND ALL THE COMPONENTS OF EXERCISE?}

Currently, the physical therapist is recognized for expertise in the treatment of the patient with a neurological disorder or post-surgery. The profession has not demonstrated its role in physical activity in individuals without an underlying medical or pain condition. The profession has not conveyed the complexity of optimizing physical activity and how individual characteristics including structural variations need to be considered. Embracing the movement system to promote the identity of physical therapy would be a step in demonstrating such expertise. Changing the longestablished pattern of a limited number of treatments for an episode of care, for what was considered an acute problem, to an expanded role that includes prevention, program development, and on-going monitoring is challenging but consistent with the chronic nature of health conditions. Such change would substantially increase the demand for physical therapists. The challenges to the current practice and business models indicate that change from the established pattern is not only the right thing but also the necessary thing to do.

One indicator of these challenges is that, instead of strong professional growth, a report from an American Physical Therapy Association (APTA) workforce analysis indicated that there may be a 25,000 oversupply of therapists by $2030 .{ }^{7}$ Another indicator is that the profession is being frequently challenged by actions to reduce reimbursement. There are other factors indicating the profession must clarify its identity for long-term viability. Demands for increased productivity is enough of a concern to spur House of Delegates action. ${ }^{8}$ In addition, the requirement of doctoral level education has not resulted in recognition commensurate with the title, or of the implied movement or exercise expertise, or a substantial increase in direct access patients/clients. Unwarranted variability in practice compromises the demonstration of value for physical therapy interventions. Why hasn't the recognition or identity of the profession progressed to take advantage of the new understanding of health conditions and the importance of movement? Have we clarified how the title of doctor is appropriate and how we are movement experts? Transforming our identity from therapists implementing treatment to doctors of an important body system, the movement system, would contribute to recognition and growth.

\section{ACTIONS THAT HAVE BEEN TAKEN AND ACTIONS THAT NEED TO BE TAKEN}

In 2013, eight years ago, the APTA initiated activities that could clarify and address the challenges to expanded recognition. These activities, 1) an outward looking vision statement, 2) a movement system identity, 3) a movement screening exam, and 4) development of specific movement 
related diagnoses, were to build on the many progressive actions that have taken place since 1979 and that were highlighted in Vision 2020. In 1979 the APTA required the entry professional degree to be beyond the baccalaureate level. Ultimately in 2018, 39 years later, the clinical doctorate became the required educational degree. ${ }^{9}$ Many, APTA documents refer to physical therapists being doctors, diagnosticians, ${ }^{10}$ movement experts, providing direct access, ${ }^{11}$ and using evidence-based practice. But these roles of physical therapists have limited recognition because the expected details and abilities associated with these roles have not been defined.

For example, although most physical therapists are doctors they do not demonstrate characteristics of this level of autonomous practitioners. Doctors are recognized for their expertise in a body system and for making a diagnosis of conditions affecting that system. Though numerous APTA documents state that physical therapists are diagnosticians, ${ }^{10}$ there is not one document that provides any examples of the diagnoses that physical therapists make. Without demonstrated expertise in a body system, diagnostic ability, or role as an autonomous practitioner, the implementation of direct access is limited. Currently expertise is based on providing treatment for a condition that another health care practitioner diagnoses. The 2013 vision statement that physical therapy will "transform society by optimizing movement to enhance the human experience" 12 requires recognition of movement expertise. The profession has not officially defined what constitutes movement expertise nor how we can or do optimize movement or even what is meant by optimizing movement.

The insightful members of the APTA committee charged to develop the 2013 Vision statement addressed implementation by designating eight guiding principles accompanying the vision statement. ${ }^{12}$ Recognizing the long-standing concerns of professional identity, ${ }^{13-15}$ the first guiding principle is that the movement system is the identity of physical therapy. The importance of this identity is reflected in the accompanying statement:

\footnotetext{
"Physical therapy will define and promote the movement system as the foundation for optimizing movement. The recognition and validation of the movement system is essential to fully understand the physiological function and potential of the human body."
}

These are two powerful statements that clearly make the point that professional recognition requires promotion of expertise in the movement system. Our professional expertise should include understanding 1) how movement causes or exacerbates problems, especially musculoskeletal (kinesiopathology), 2) how movement becomes dysfunctional with pathology in component systems (pathokinesiology), 3 ) how the result is compromised function, health, and/or quality of life, and 4) how movement in the form of activity or exercise is a powerful prevention or moderator of disease. The profession needs to clarify that therapists not only have expertise in dose and type of exercise during rehabilitation but also in prevention and reduction of injury resulting from exercise and activity in individuals without an identified problem.

Recognition and implementation of the profession's role in healthcare should be achieved in the same way as other health professions have achieved recognition by demonstrating expertise in a body system and using recognized labels for potential or actual diagnoses. Doctors are consulted for a diagnosis or a potential problem, that conveys to the patient and other health professionals, knowledge of dysfunctions of a body system. The diagnosis is also the guide to treatment. The question is whether the profession can take 39 years to implement the actions required for recognition of doctoral level education and practice.

\section{IMPLEMENTATION OF THE MOVEMENT SYSTEM IDENTITY}

\section{INITIAL STEPS}

In 2013 after acceptance of the vision statement and guidelines, a Board of Directors Work Group was appointment to develop a definition of the Movement System and a Plan of Implementation. The eight members including three individuals from the APTA board of directors, were educators, practitioners, and researchers. A white paper was produced and published in 2015 on the APTA website that included the following definition and statements of physical therapists' role in the movement system:

"The human movement system (HMS) comprises the anatomic structures and physiologic functions that interact to move the body or its component parts.

\section{PHYSICAL THERAPIST PRACTICE AND THE MOVEMENT SYSTEM}

Human movement is a complex behavior within a specific context.

Physical therapists:

- Provide a unique perspective on purposeful, precise, and efficient movement across the lifespan

- Based upon the synthesis of their distinctive knowledge of the movement system and expertise in mobility and locomotion.

- Examine and evaluate the movement system (including diagnosis and prognosis)

- Provide a customized and integrated plan of care

- To achieve the individual's goal directed outcomes.

- Maximize an individual's ability to engage with and respond to their environment

- Using movement related interventions to optimize functional capacity and performance.

By further defining and articulating PT's unique role in diagnosing and managing both risk of and intervention for movement system disorders:

- We professionally advance toward standardizing our management methods to reduce unwarranted variations in practice and

- Subsequently enhance our ability to achieve consistent positive outcomes for specific diagnoses and

- Enhance the value of the services we provide. 


\section{A DETAILED PLAN FOR ACHIEVING THESE GOALS WAS ALSO DEVELOPED.}

The white paper also described 1) the basic requirements for a human movement system practitioner, 2) how the movement system identity can impact practice and education, and 3) implications for research. The impact of identity on practice was stated as: "A movement system practitioner, the PT has the expertise to examine, diagnose, and treat all elements of this system to produce a meaningful change in an individual's movement behavior and physical function. The PT uses his or her integrative knowledge to establish a plan of care to maximize physical performance of people of all ages, pathologies, or levels of physical function.”

The paper further articulates the relevance of the human movement system (HMS) to society and the profession by stating that “...promotion of the physical therapists' expertise in diagnosing and treating movement impairments will have profound impact on the health of the public" by:

1. Promoting a more active population,

2. Returning patients/clients to participation in life, and

3. Reducing the consumption of unnecessary, high-cost medical procedures.

To make this a reality, the profession must develop a common language to describe our diagnostic process and our management of the HMS. A major impact of a common approach to detailing the HMS is that communities internal and external to the profession will communicate via a common language that accounts for the complexity of optimal movement.

These are insightful and powerful statements that make the case for why promotion of the HMS is important to the recognition and growth of the profession. Unfortunately, the limited distribution and implementation of the recommendations have not had the impact that is necessary for recognition of the HMS and thus have not successfully expanded the role of the profession.

Another important action reinforced the recommendations of the work group. In 2015 the House of Delegates adopted the motion, "Resolved, [the] APTA endorses the development of diagnostic labels and/or classification systems that reflect and contribute to the physical therapists' ability to properly and effectively manage disorders of the movement system." 16

After completion of their charges by the work group, a movement system task force was appointed to further develop and implement the recommendations. The nine members included two individuals from the Board of Directors. These members were also educators and practitioners. One of the actions recommended by the task force was to conduct a Movement System Summit. One hundred individuals were invited to participate in the summit including a representative from all of the academies and sections. The major goals of the summit, held in 2016, were to discuss 1) what is our understanding of the movement system, 2) what essential components should be included in our examination, and 3) what is a movement system diagnosis?

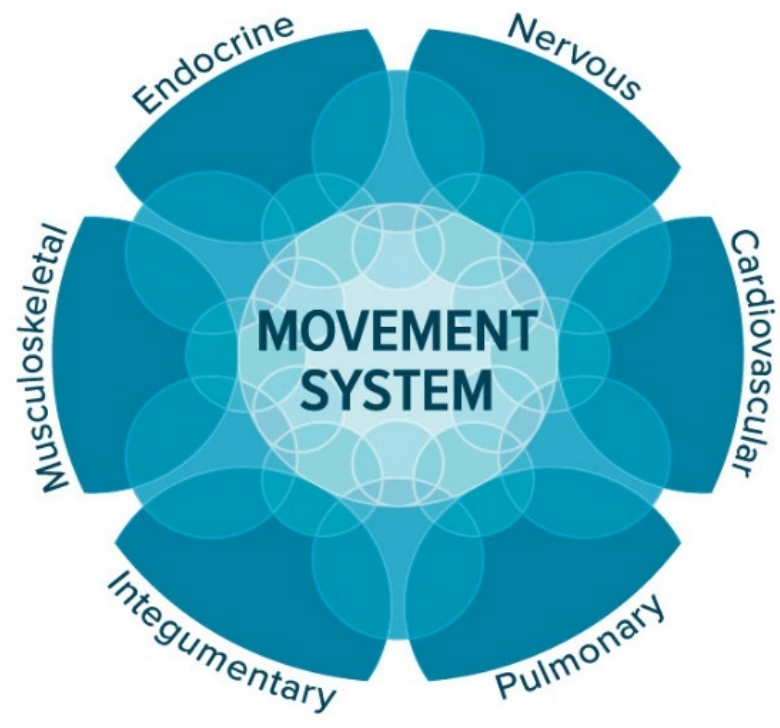

\section{THE HUMAN MOVEMENT SYSTEM}

A graphic depicting the major systems composing the movement system was developed. Though other physiological systems can affect movement, these systems were considered to have the major effects on movement.

A revised definition of the movement system was adopted by the House of Delegates in 2019.10

The official definition is "The integration of body systems that generate and maintain movement at all levels of bodily function.”

\section{MOVEMENT SCREENING EXAM}

There was consistent agreement at the summit that assessment of basic body movements and mobility tasks should comprise the examination across settings and practice areas of physical therapy. The purpose of this screen is to be "a tool designed to detect movement impairments observed during functional task/activities that will help therapists decide which additional tests and measures to include in the patient examination." Subsequently meetings with representatives of the Academies and Sections of the APTA were held to further develop and implement the screening examination. The content of the screening examination was considered complete enough to develop a form and general instructions that could be distributed for piloted use. A form with instructions has been appended to this commentary (Appendix A). This screen can provide some commonality to a basic physical therapy assessment that would inform other health care practitioners of our expertise as well as baseline information about the patient. Consistent intake information would convey to others how physical therapists assess the major aspects of movement performance. Additional guidelines regarding decisions related to what is considered impaired depending upon the complaint and severity of the patient's condition would be useful. In other words, impaired performance of a patient with a muscu- 
loskeletal problem would likely be more subtle than the impairment of a patient with a neurological disorder.

\section{MOVEMENT SYSTEM DIAGNOSES}

In 2017, the APTA Board of Directors adopted a set of criteria to be used by any stakeholder groups developing diagnostic systems/labels that had been discussed and recommended at the summit. The recommended criteria are:

1. Use recognized movement-related terms to describe the condition or syndrome of the movement system.

2. Include, if necessary, the name of the pathology, disease, disorder, anatomical or physiological terms, and stage of recovery associated with the movement diagnosis.

3. Be as succinct and direct as possible to improve clinical usefulness.

4. Strive for movement system diagnoses that span all populations, health conditions, and the lifespan. Whenever possible, use similar movement-related terms to describe similar movements, regardless of pathology or other characteristics of the patient or client.

At a subsequent meeting with representatives of the academies and sections designed to further identify criteria for developing diagnoses, the decision was made to solicit diagnoses from the membership and a form for that purpose was developed (Appendix A). Ninety suggested diagnoses were received from the membership. There was also a recommendation for the academies and sections to continue with the work of developing diagnoses.

\section{CURRENT STATUS AS OF NOVEMBER 2021}

Additional implementation of the movement screen and diagnosis development was assigned to the APTA practice and science committee. The process has stalled. No additional information about the movement system, the movement screen, or movement-related diagnoses has been provided by the APTA. It would seem that this is an opportune time as we as a profession consider how physical therapy will be positioned for the next 100 years.

Early in the process the former Section on Women's
Health developed diagnoses but after the publication of the initial work, ${ }^{17}$ nothing additional has been published. The Academy of Neurological Physical Therapy has made major efforts to develop and promote movement system diagnoses. The Academy of Neurological Physical Therapy has formed several task forces and a white paper has been published. ${ }^{18} \mathrm{~A}$ recent publication detailed the diagnoses that have been developed for balance ${ }^{19}$ and a document to guide task analysis has also been published. ${ }^{20}$ The Academy of Pediatric Physical Therapy has joined with the Academy of Neurologic Physical Therapy to continue the development of diagnoses.

The International Journal of Sports Physical Therapy once again has committed to publishing a special issue on the Movement System. The initial publication on the topic in 2017 contained many key articles and offered insightful perspectives. The topic is an important one. In this era where distribution of ideas and actions can be spread widely and rapidly, the APTA membership can play a significant role in implementing change. The membership will be affected in the long run by how the profession is recognized for its value to health care. There is every reason to promote the movement system in conversations with both patients and other health care practitioners. The point has been made in many documents that the profession needs to be valued for what we KNOW and not just what we do.

My hope is that in furthering the professionalization of physical therapy by recognized expertise in a physiological body system and use of diagnostic labels there could also be action to change the name of the practitioner. To clearly recharacterize our role in health care and be consistent with being doctors, the label of therapist needs to be changed. Speech therapy has made this change without an educational change to the doctoral level. They are now speech and language pathologists. The label pathologist conflicts with a role in prevention but a label using "ologist" would be consistent with other health care practitioners. However, the name of the profession does not need to change but an upgrade to the title of the practitioner to clarify that the next 100 years will be for growth in responsibility, recognition, and demand may propel the profession forward in an amazing way.

Submitted: November 01, 2021 CST, Accepted: November 28, 2021 CST 


\section{REFERENCES}

1. National Center for Chronic Disease Prevention and Health Promotion. https://www.cdc.gov/chronicdiseas e/about

2. Barrett JP, Olivari BS, Price AB, Taylor CA. Cognitive decline and dementia risk reduction: Promoting healthy lifestyles and blood pressure control. Am J Prev Med. 2021;61(3):e157-e160. doi:1 0.1016/j.amepre.2021.03.005

3. Hojman P, Gehl J, Christensen JF, Pedersen BK. Molecular mechanisms linking exercise to cancer prevention and treatment. Cell Metab. 2018;27(1):10-21. doi:10.1016/j.cmet.2017.09.015

4. Stout NL, Baima J, Swisher AK, Winters-Stone KM, Welsh J. A Systematic review of exercise, systematic reviews in the cancer literature (2005-2017). PMR. 2017;9(9S2):S347-S384. doi:10.1016/j.pmri.2017.07.07 $\underline{4}$

5. Lamb SE, Sheehan B, Atherton N, et al. Dementia and physical activity (DAPA) trial of moderate to high intensity exercise training for people with dementia: A randomised controlled trial. BMJ. 2018;361:k1675. doi:10.1136/bmj.k1675

6. Gholamnezhad Z, Boskabady MH, Jahangiri Z. Exercise and dementia. Adv Exp Med Biol. 2020;1228:303-315. doi:10.1007/978-981-15-179 $\underline{2-120}$

7. The American Physical Therapy Association. APTA Work Force Analysis Published 12/2020.

8. The American Physical Therapy Association. APTA 2021 House of Delegates - 2021 House of Delegates Recap The association's representative body of the membership addressed racism, DEI, PTA involvement, COVID-19, productivity standards, and more.

9. The American Physical Therapy Association. Educational Degree qualification and nomenclature for physical therapists and physical therapists assistant. HOD P06-18-33-38.

10. The American Physical Therapy Association. Diagnosis by Physical Therapists: HOD P06-2-10-09 Amended HOD P06-08-06-07; HOD P06-97-06-19; HOD 06-95-12-07; HOD 06-94-22-35; Initial HOD 06-84-19-78].
11. The American Physical Therapy Association. Physical Therapists as Primary Care and Entry-Point Providers. HOD P06-18-28-22.

12. The American Physical Therapy Association. Vision Statement for the Physical Therapy Profession HOD P06-13-18-22.

13. Hislop HJ. Tenth Mary McMillan lecture: the notso-impossible dream. Phys Ther. 1975;55(10):1069-1080. doi:10.1093/pti/55.10.1069

14. Rothstein JM. Pathokinesiology: a name for our times? Phys Ther. 1986;66:364-365.

15. Sahrmann S. The human movement system, our identity. Phys Ther. 2014;94(7):1034-1042. doi:10.252 2/pti.20130319

16. Management of the Movement System HOD P06-15-25-24.

17. Spitznagle T, Cabelka C, Clinton S, Abraham K, Norton B. Diagnosis dialog for women's health conditions: The process and proposed pelvic floor muscle diagnosis. J Womens Health Phys Ther. 2017;41(3):154-162. doi:10.1097/jwh.0000000000000 $\underline{086}$

18. Quinn L, Riley N, Tyrell CM, et al. A framework for movement analysis of tasks: Recommendations from the academy of neurologic physical therapy's movement system task force. Phys Ther. 2021;101(9). doi:10.1093/pti/pzab154

19. Hedman LD, Quinn L, Gill-Body K, et al. White paper: Movement system diagnoses in neurologic physical therapy. J Neurol Phys Ther. 2018;42(2):110-117. doi:10.1097/npt.00000000000002 15

20. Gill-Body KM, Hedman LD, Plummer L, et al. Movement system diagnoses for balance dysfunction: Recommendations from the academy of neurologic physical therapy's movement system task force. Phys Ther. 2021;101(9). doi:10.1093/ptj/pzab153 


\section{SUPPLEMENTARY MATERIALS}

\section{Appendix A}

Download: https://iispt.scholasticahq.com/article/30175-doctors-of-the-movement-system-identity-by-choice-ortherapists-providing-treatment-identity-by-default/attachment/76690.docx?auth token=eyq6BfCE47iaKMgWsacd 